\title{
Automatic Verification of Heap Manipulation Using Separation Logic
}

\author{
Josh Berdine
}

Microsoft Research

Accurately representing the structure and manipulation of heap-allocated data structures is a key challenge for automatic verification of nontrivial code. Capturing this information is central to proving properties such as the absence of invalid memory accesses (for instance, deallocating memory twice or dereferencing dangling or NULL pointers), the absence of memory leaks, and data-structure integrity properties (for instance, that a doubly-linked list structure is properly linked in both directions).

Significant advances in this area have recently been made by using separation logic for the abstract representation of program states and semantics of commands. Here we explain the core of the approach that has enabled these advances in the automatic verification of deep heap properties, without requiring programs to be annotated by the developer.

Additionally, starting from this core for verification of partial correctness properties, we present new techniques for termination analysis of heap manipulating code that are based on generating an abstraction for termination from a proof of partial correctness. This results in a purely arithmetic abstraction of heap manipulating code, which enables recent advances in termination proving to be applied to code that operates not only on integer data, but also on heap-allocated data structures. 